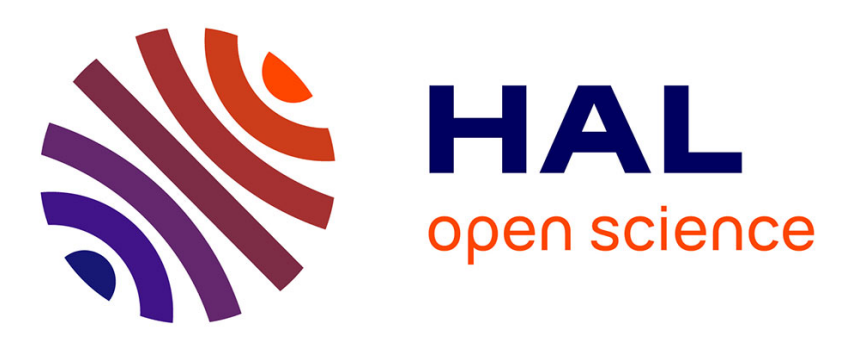

\title{
Automatic Re-synchronization Controller Analysis within a Multi-Domain Gas Turbine and Power System Model
}

Luigi Vanfretti, Biswarup Mukherjee, Kannan M Moudgalya, Francisco J Gómez

\section{To cite this version:}

Luigi Vanfretti, Biswarup Mukherjee, Kannan M Moudgalya, Francisco J Gómez. Automatic Resynchronization Controller Analysis within a Multi-Domain Gas Turbine and Power System Model. 2019 7th Workshop on Modeling and Simulation of Cyber-Physical Energy Systems (MSCPES), Apr 2019, Montreal, Canada. pp.1-5, 10.1109/MSCPES.2019.8738797 . hal-03297098

\section{HAL Id: hal-03297098 \\ https://hal.science/hal-03297098}

Submitted on 23 Jul 2021

HAL is a multi-disciplinary open access archive for the deposit and dissemination of scientific research documents, whether they are published or not. The documents may come from teaching and research institutions in France or abroad, or from public or private research centers.
L'archive ouverte pluridisciplinaire HAL, est destinée au dépôt et à la diffusion de documents scientifiques de niveau recherche, publiés ou non, émanant des établissements d'enseignement et de recherche français ou étrangers, des laboratoires publics ou privés. 


\section{Automatic Re-synchronization Controller Analysis within a Multi-Domain Gas Turbine and Power System Model}

\author{
Luigi Vanfretti ${ }^{1}$ \\ ECSE \\ Rensselaer Polytechnic Institute \\ Troy, NY, USA \\ vanfrl@rpi.edu
}

\author{
Biswarup Mukherjee ${ }^{2}$, Kannan M. Moudgalya \\ Department of Chemical Engineering, FOSSEE \\ Indian Institute of Technology Bombay \\ Mumbai, India \\ bismuk.ece@gmail.com, kannan@iitb.ac.in
}

\author{
Francisco J. Gómez \\ Semantum $O y$ \\ Espoo, Finland \\ francisco.gomez@semantum.fi
}

\begin{abstract}
This paper presents the design of a centralized controller architecture for automatic re-synchronization of islanded networks that uses remote synchrophasor measurements from Phasor Measurement Units (PMUs). The controller behaviour is tested in a multi-domain power system model, where a thermomechanical model of a gas turbine is used within the controlled generator to model a Distributed Energy Resource (DER), in substitution of a traditional turbine-governor representation. The controller architecture uses PMU data from substations at transmission and distribution level. Considering different power dispatch levels of the distribution generator model in the power system, the performance of frequency control module inside the overall re-synchronization controller has been analyzed and compared for both electrical-domain and multi-domain models. This paper shows that multi-domain models provide more detailed representation of the turbine behaviour and a better adjustment of the control signal behavior during the resynchronization process.

Index Terms-Automatic re-synchronization controller; power grid; power systems; distribution network; synchrophasors; Modelica; OpenIPSL; ThermoPower; gas turbines; multi-domain modeling and simulation
\end{abstract}

\section{INTRODUCTION}

\section{A. Motivation and Literature Review}

With the increasing number of intermittent Distributed Energy Resources (DERs), frequency control in power grids is becoming increasingly difficult. In any islanded portion of a micro-grid, reference [1] proposes the use of electronically interfaced distributed generators (EI-DG) units that should take part in frequency and voltage control along with synchronous generator based DG units to assure micro-grid power quality and stability. Conventionally, transmission system operators (TSOs) and power plants often need to coordinate the resynchronization process of any islanded portion of the power grid so that the proper balance between supply and demand can

Authors contributed equally for this work ${ }^{1,2}$.

L. Vanfretti is supported in part by the Engineering Research Center Program of the National Science Foundation and the Department of Energy under Award EEC-1041877 and in part by the CURENT Industry Partnership Program.

B. Mukherjee and K. M. Moudgalya are supported by MHRD, Government of India, FOSSEE-Phase II and Spoken Tutorials Projects at IIT Bombay. be met. It is reported in [2] that conventional synchronization techniques fail to maintain the power system stable when disturbances occur during the re-synchronization process and, in addition, improper re-synchronization might degrade the power quality, reliability and security of the power transmission system [3].

To address issues related to improper re-synchronization, reference [4] presents the modeling of an automatic resynchronization control architecture to re-synchronize an isolated distribution network with DER by using measurement data from Phasor Measurement Units (PMUs), located at transmission and distribution substations. This re-synchronization method is attractive for the low voltage distribution grids due to increased penetration of DERs. The multi-domain modeling approach was discussed and suggested for the modeling of the power grid in [5] [9] validates the idea to use Modelica language for complex energy systems modeling without any loss of information, however, that work does not report any control system design for turbine and governor system. The work presented in [5] also shows that the multi-domain modeling approach is essential for power system analysis when gas turbines are used to provide grid flexibility and this is especially important for controller system design. The work presented by the authors in [4] reports the re-synchronization control architecture used herein, which was analyzed only using component models from the power system domain. Hence it is of interest to study the performance of the control system design when exposed to a multi-Domain gas turbine and power system model. Therefore this paper applies the multi-domain modeling approach to the design and implementation of an automatic re-synchronization control system using the Modelica language [6]. The ThermoPower library [7] is utilized to represent the thermo-mechanical dynamics of the gas turbine, and the OpenIPSL library [8], is used to model the rest of the power system components.

\section{B. Paper Contributions}

This paper studies the sequential re-synchronization control architecture in both conventional power system and multi- 


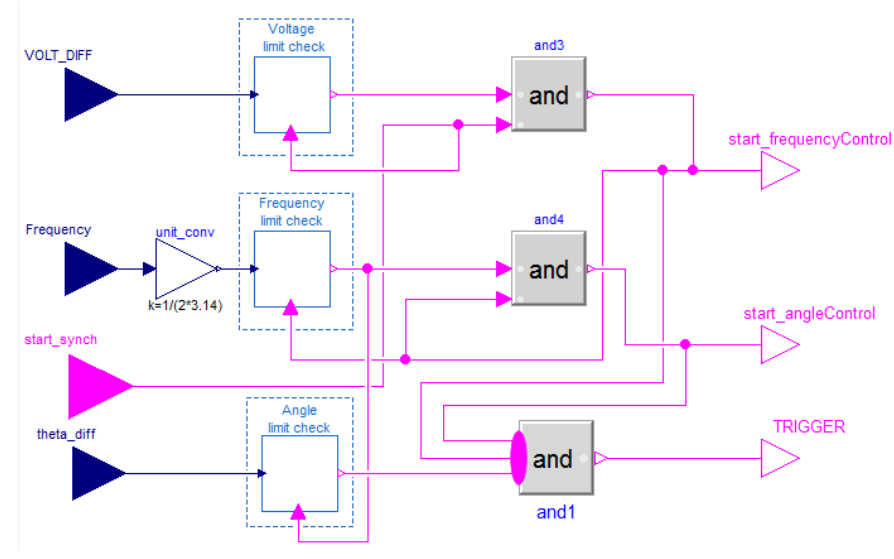

Fig. 1. Sequential control mode implementation in Modelica

domain models that includes gas turbine dynamics coupled to those of the power grid. One of the tasks within the ITEA3 OpenCPS (Open Cyber-Physical System Model-Driven Certified Development) project [10] was to develop Modelica models for automatic re-synchronization and islanded operation controller in electrical power systems.

The authors developed benchmark power system models for the OpenCPS project to test and validate results for the re-synchronization controller that exhibits how controllers in a power grid can be modeled to incorporate the dynamic behavior of a gas turbine and governor system modeled from the thermo-mechanical point-of-view and not only the conventional power system domain. Therefore this paper reports extended modeling control design and performance assessment of the re-synchronization controller using the multi-domain modeling approach, which was previously reported by the authors in [4] only within the power system domain.

\section{Controller Modeling in a Multi-domain Power SYSTEM MODEL}

\section{A. The automatic re-synchronization controller}

The Modelica implementation of the automatic resynchronization controller was first proposed in [4]. This controller uses a sequential control mode that is implemented inside an activationUnit. The purpose of this control mode is to activate three individual controllers (i.e. firstly a voltage difference controller, next frequency difference controller and finally an angle difference controller) in a 'sequence'. Fig. 1 shows the Modelica implementation, while the algorithm implemented for the sequential control mode is presented in Fig. 2.

The LimitCheckTriggered blocks inside the activationUnit perform checking of individual thresholds for each of the synchronization variables $(\Delta \mathrm{V}, \Delta \mathrm{f}$ and $\Delta \theta$ ) before completion of each step (i.e. Stages 1, 2 and 3 ) inside the implemented control algorithm presented in Fig. 2. Therefore the output of this LimitCheckTriggered block should be true if the input to the block lies within the thresholds during dt seconds, as shown in the Modelica

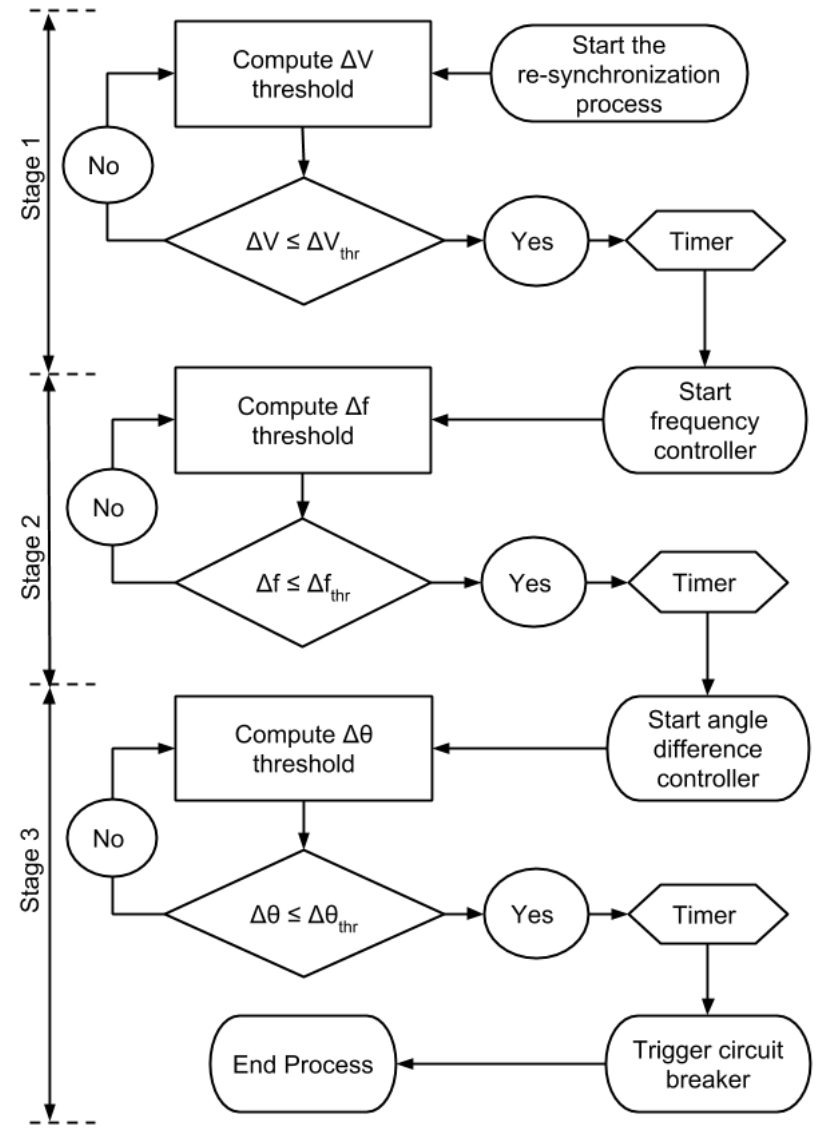

Fig. 2. Algorithm of the sequential control mode

code listing below. Detail modeling and operation of this LimitCheckTriggered block are beyond the scope of this paper, and will be discussed in a future publication.

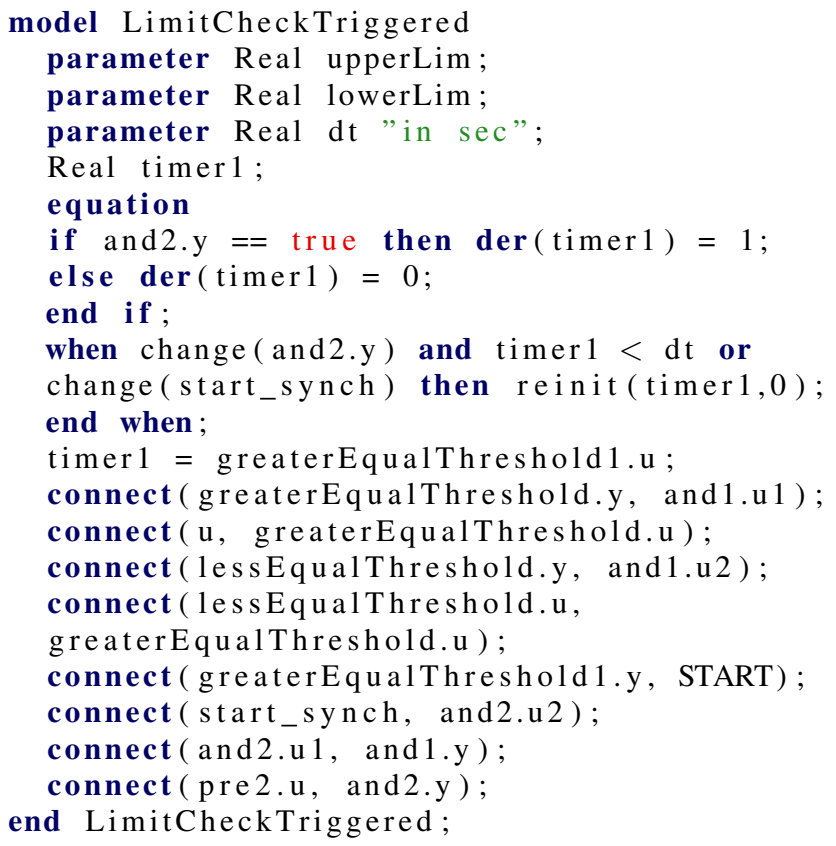




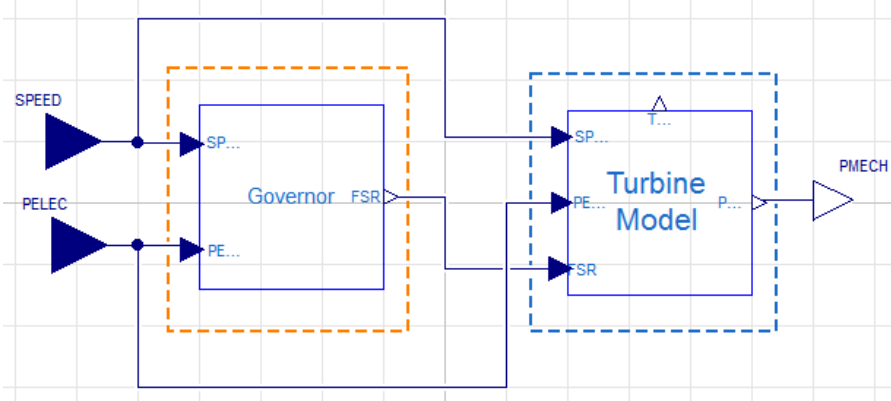

Fig. 3. Turbine model separation from GGOV model implementation in OpenISPL

\section{B. Multi-Domain Modeling}

For the power system-only model, the (GGOV1) shown in Fig. 3 represents separately a governor model, in addition to a block which represents a conventional gas turbine model. The turbine model requires speed, electrical power and the governor's output to calculate the desired output mechanical power. Meanwhile, in the multi-domain model in [5], the turbine model output is mechanical power (Pmech) and the input is the fuel flow $(W F)$. In steady-state, the mechanical power produced by the turbine is therefore described by

$$
P_{\text {mech }}=K_{\text {turb }} \times\left(W F-W_{f n l}\right)
$$

where $W f n l$ is the constant fuel flow at maximum speed, and $K_{\text {turb }}$ is the turbine gain which affect the behaviour of GGOV1.

Reference [5] describes in detail the differences between the simple turbine model (GGOV1) and multi-domain model. The multi-domain model is composed of a single shaft gas turbine using components from from ThermoPower and a governor model from the OpenIPSL. As the power system model of the turbine is composed of simplified transfer functions, it excludes important dynamics of the actual turbine system that may be excited during the re-synchronization process due to its long time-scale. Using the features of ThermoPower library the turbine dynamics can be modeled to improve the design and performance of the automatic re-synchronization controller. Figure 4 shows the generator model implemented in Modelica, that includes the turbine model developed using ThermoPower. The gas turbine model produces the mechanical power that can be determined from the following equation:

$$
P_{\text {mech }}=\omega \times \tau
$$

where $\omega$ is the rotational speed and $\tau$ is the shaft torque. This equation has been used inside the interface model TM2EPConverter that transforms the turbine mechanical power to the electrical power using the Modelica code listing below [14].

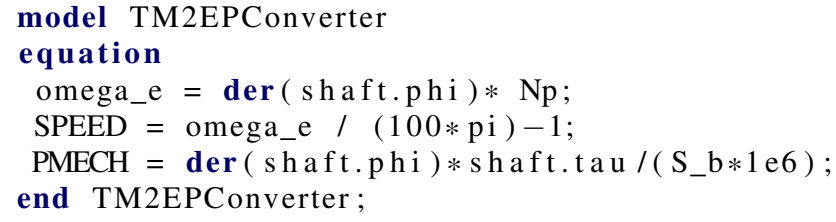

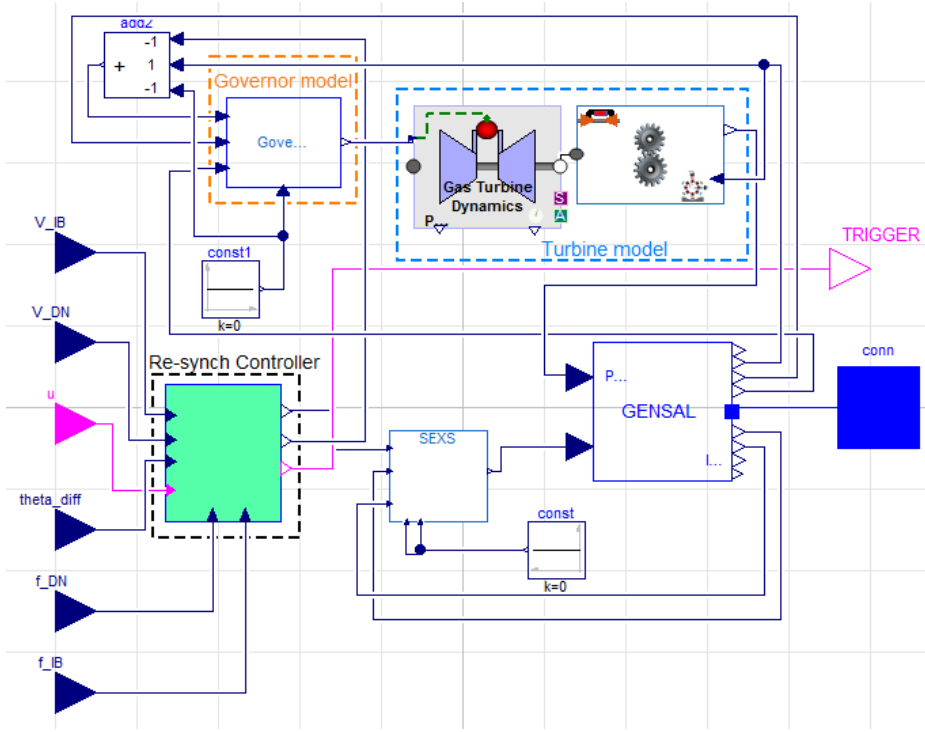

Fig. 4. Centralized control structure within the generator model (G22) including gas turbine model built using ThermoPower.

\section{Power System Model and Simulation Set-uP}

The power system model implemented in Modelica to study the performance of the automatic re-synchronization controller is shown in Fig. 5. The controller model is implemented inside the DER generator at the distribution network, which was presented in the previous section. The 'Record' shown in Fig. 5, enables the use of Modelica records [12] to store data in a systematic way and to propagate it for different scenarios. These replaceablePowerflowData records contain the initial guess values of the power flow solution obtained from the PSS/E software [13], which are obtained after running a Python script that generates Modelica records from a . raw file. This allows to evaluate the controller's performance at multiple power dispatch levels.

The simulation set-up block implemented in Modelica is presented in Fig. 6, it can be seen as a process to create simulation scenarios consistently. It is used to create the sequence of actions that are necessary to evaluate the automatic re-synchronization controller. In this sequence, circuit breaker 1 is controlled implementing the following logic. For the first 6 seconds, the breaker remains closed and after that it is opened thereby islanding the distribution network where DER is placed. At 6.01 seconds the re-synchronization process starts (as it is intended to activate the automatic re-synchronization controller as fast as possible after the distribution grid is islanded) and the Boolean signal y3 becomes true. This output is applied as the Boolean input to the automatic resynchronization unit to start the its control sequence. When the Boolean output from the activating unit becomes true, the breaker CB2 is closed to re-synchronize the islanded distribution network with the transmission grid. The outputs y 1 and $y 2$ represent the measurements from the PMUs that are used by the controller inside the generator model G22. 


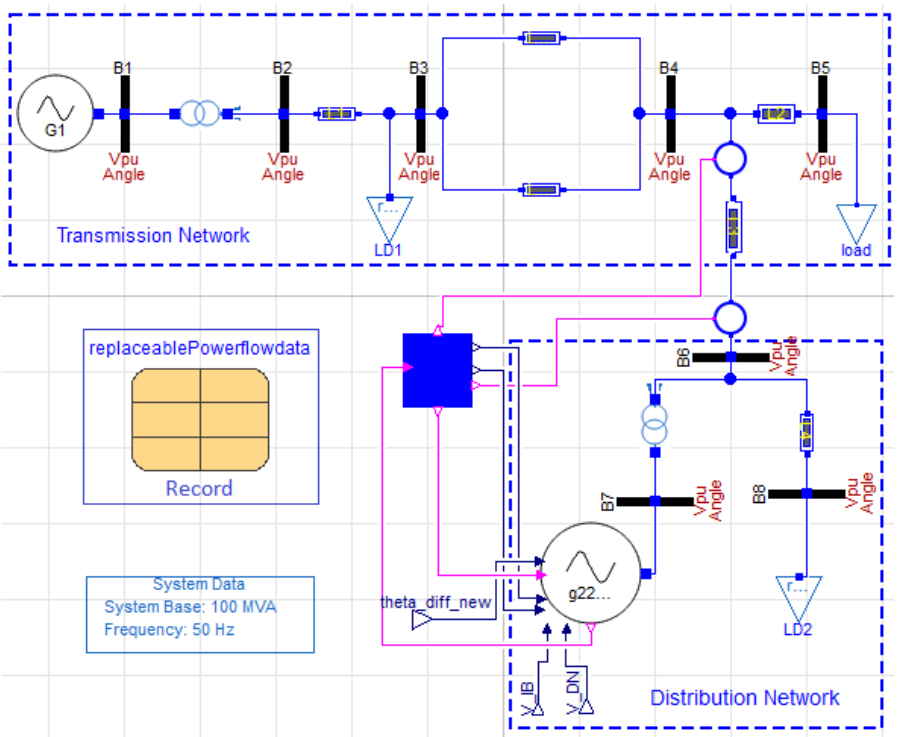

Fig. 5. Power system model

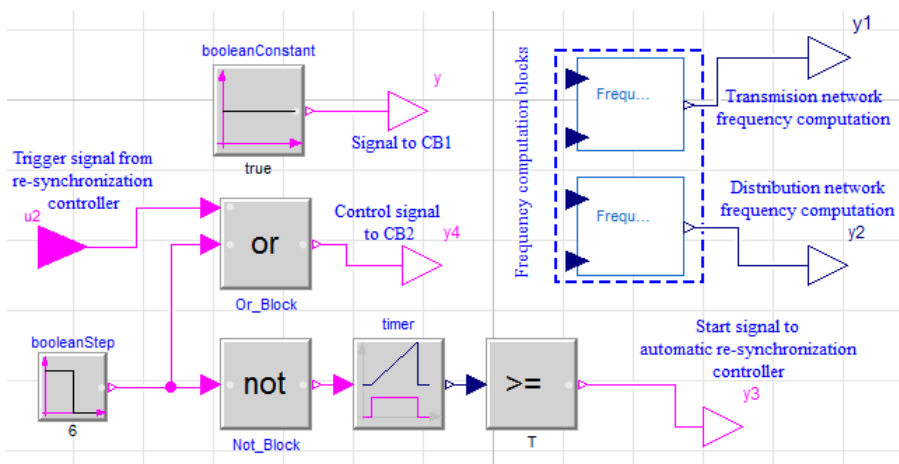

Fig. 6. Modelica implementation of the simulation set-up.

The circuit breaker 2 remains constantly closed to keep the line (L3) energized from the transmission network.

\section{Simulation Results}

This section discusses the performance analysis of the automatic re-synchronization controller in the multi-domain power system model. Simulation results are plotted in Fig. 7 and Fig. 8, and show the output of the frequency controller (which is a PI controller within stage 2 of Fig. 2) inside the automatic re-synchronization controller. From Fig. 7 observe that with the increase of the power dispatch (i.e. from 5MW to $10 \mathrm{MW}$ ) from the distribution DER the peak overshoot of the frequency controller's output increases.

Figure 8 shows the frequency controller output for different dispatch levels both in power system-only and multidomain models. It is interesting to observe that, as the resynchronization controller model is subjected to the gas turbine dynamics, the control loop takes longer time to reach a new steady state as compared to power system-only model.

Figure 9 plots the settling time of the frequency controller output for both power system-only and multi-domain control

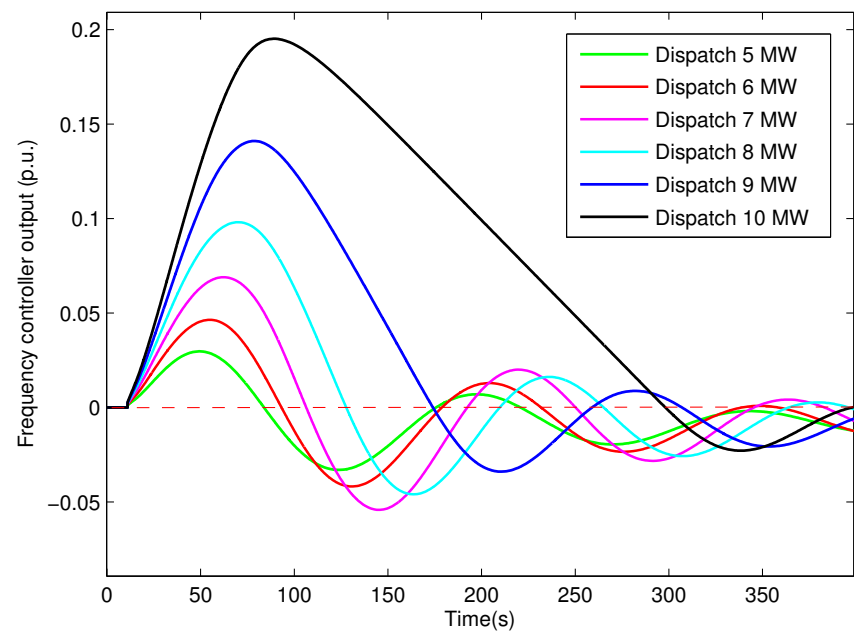

Fig. 7. Frequency controller output for different dispatch levels from the distribution generator.

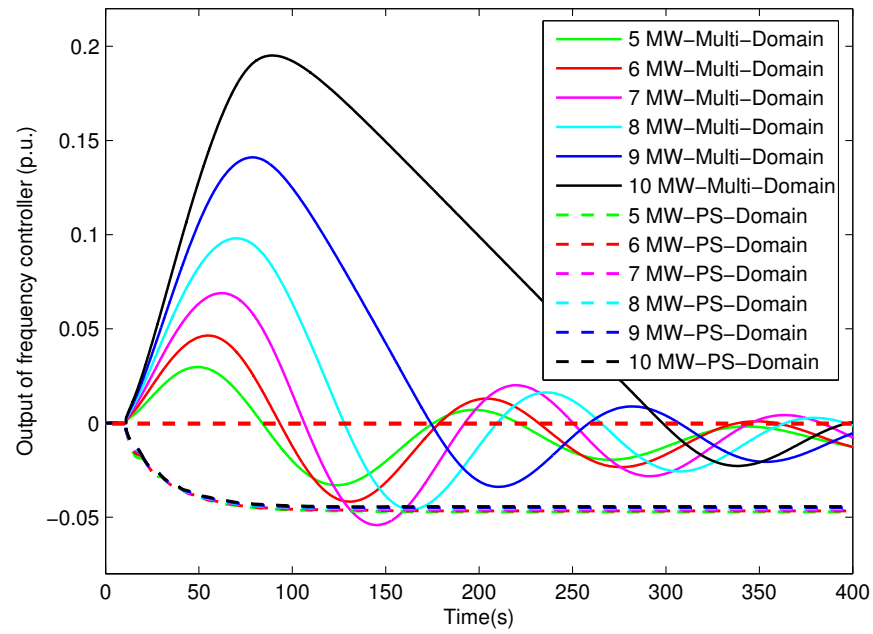

Fig. 8. Comparison of frequency controller output in the multi-domain and power system domain models.

architectures. It can be observed from Fig. 9 that in the case of multi-domain model it takes significantly longer time for the control loop to settle, so as to activate the angle difference controller, resulting in a longer time to safely close the circuit breaker CB2. Observe in Fig. 9 that the frequency error for the multi-domain model also does not return to zero when the distribution network is islanded, this is because there is no mechanism or controller to set the synchronous speed, and therefore, the steady-state frequency when islanded is set by the minimum deviation allowed by the governor's droop. This can be avoided by using an islanded operation controller as proposed in [11], which will be investigated in the future within the multi-domain setting. This analysis is extended for multiple dispatch levels in Fig. 10, where the DER is 


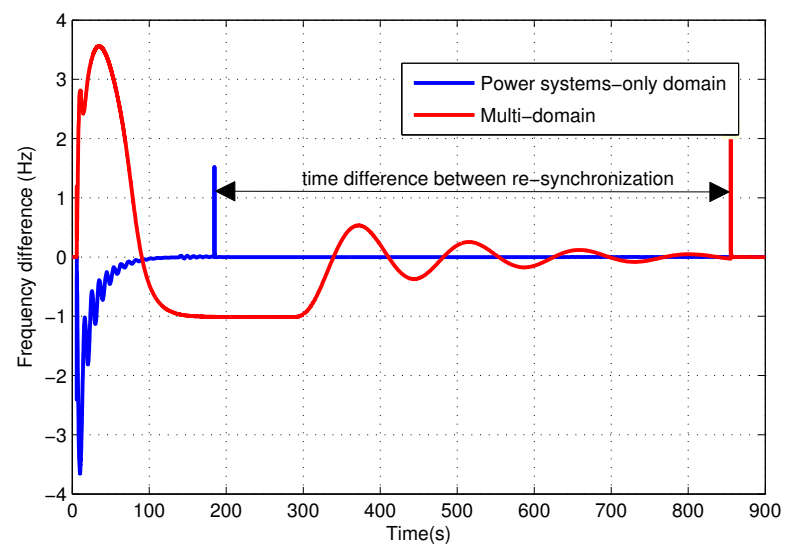

Fig. 9. Comparison of frequency difference both in multi-domain and power system-only models.

set to dispatch from $5 \mathrm{MW}$ to $10 \mathrm{MW}$. The figure shows that it takes on average 700 additional seconds to minimize the frequency difference in order to safely re-synchronize the network. This clearly shows the importance of multi-domain modeling for processes such as this, where long time constants of thermo-mechanical processes cannot be neglected as they are intricately involved in meeting the control objectives.

\section{CONCLUSions And Further Work}

This study shows multi-domain approach is essential for power system analysis, especially when designing the control systems involving prime movers that involve long time scales. From the simulation results it can be concluded that the frequency difference controller in Stage 2 of the sequential control architecture has to be re-designed. The original design carried out using the conventional power system model does not help to attain adequate performance, resulting in overshoots and insufficient damping when evaluated using the detailed gas turbine model that includes dynamics with long time scales.

Further work should incorporate the tuning of the controllers inside the control system architecture so that overall performance can be improved, and to successfully cope with remote measurement data transmission impacts such as delay and packet drops.

\section{ACKNOWLEDGMENT}

The authors would like to acknowledge research group members of ALSET Lab and FOSSEE for their valuable feedback and comments on the work in this paper.

\section{REFERENCES}

[1] P. S. R. V. R. S. S. Vidyasagar and K. S. Swarup, "Discrete model predictive frequency and voltage control of isolated micro-grid in smart grid scenario," 2016 National Power Systems Conference (NPSC), Bhubaneswar, India, 19-21 Dec. 2016, pp. 1-6. doi: 10.1109/NPSC.2016.7858879

[2] N. A. Belyaev, Y. V. Khrushchev, S. V. Svechkarev, A. V. Prokhorov and L. Wang, "Generator to grid adaptive synchronization technique based on reference model," 2015 IEEE Eindhoven PowerTech, Eindhoven, 2015, pp. 1-5. doi: 10.1109/PTC.2015.7232582.

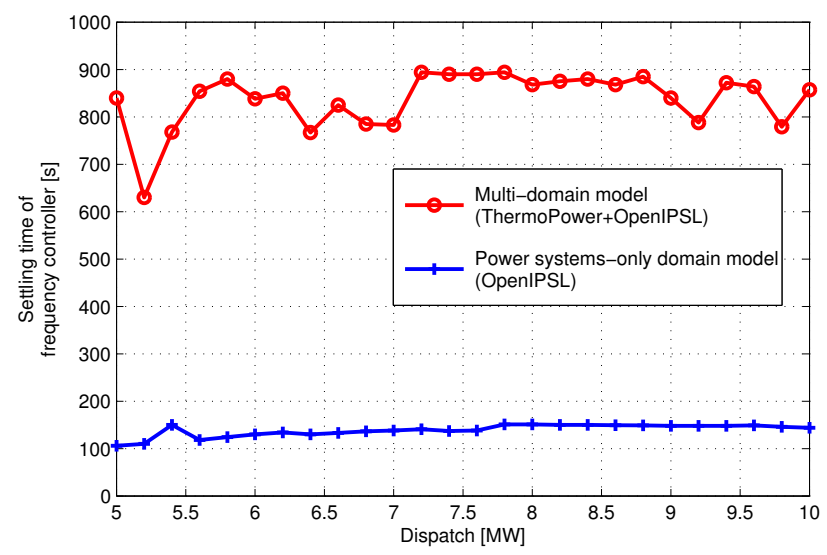

Fig. 10. Comparison of frequency controller settling time both in multidomain and power system-only models.

[3] A. Mazloomzadeh, V. Salehi and O. Mohammed, "Soft synchronization of dispersed generators to micro grids for smart grid applications," 2012 IEEE PES Innovative Smart Grid Technologies (ISGT), Washington, DC, 2012, pp. 1-7. doi: 10.1109/ISGT.2012.6175812.

[4] B. Mukherjee and L. Vanfretti, "Modeling of PMU-Based Automatic Resynchronization Controls for DER Generators in Power Distribution Networks using Modelica language," in Proceedings of the 13th International Modelica Conference, Regensburg, Germany, March 4-6, 2019, pp. 607616. doi: 10.3384/ecp19157607

[5] M. Aguilera, L. Vanfretti, T. Bogodorova and F. J. Gómez, "Coalesced Gas Turbine and Power System Modeling and Simulation using Modelica", in Proceedings of the 1st American Modelica Conference, Cambridge, Massachusetts, USA, October 9-10, 2018, pp. 138-146. doi: 10.3384/ecp 18154

[6] Peter Fritzson, "The Modelica Language," in Principles of ObjectOriented Modeling and Simulation with Modelica 2.1", IEEE, 2004, doi: 10.1109/9780470545669.

[7] F. Casella and A. Leva, "Object-Oriented Modelling \& Simulation of Power Plants with Modelica," in Proceedings of the 44th IEEE Conference on Decision and Control, Seville, Spain, 2005, pp. 7597-7602. doi: 10.1109/CDC.2005.1583388

[8] M. Baudette, M. Castro, T. Rabuzin, J. Lavenius, T. Bogodorova, L. Vanfretti, "OpenIPSL: Open-Instance Power System Library Update 1.5 to "iTesla Power Systems Library (iPSL): A Modelica library for phasor time-domain simulations", SoftwareX, Volume 7, January-June 2018, Pages 34-36, ISSN 2352-7110.

[9] M. Aguilera, L. Vanfretti and F. J. Gómez, "Experiences in power system multi-domain modeling and simulation with modelica \& FMI: The case of gas power turbines and power systems," 2018 Workshop on Modeling and Simulation of Cyber-Physical Energy Systems (MSCPES), Porto, 2018, pp. 1-6. doi: 10.1109/MSCPES.2018.8405397

[10] EU ITEA3 OpenCPS project website, Online at: https://opencps.eu

[11] B. Mukherjee and L. Vanfretti, "Modeling of PMU-Based Islanded Operation Controls for Power Distribution Networks using Modelica and OpenIPSL," in Proceedings of The American Modelica Conference, Cambridge, MA, USA, Oct. 9-10, 2018, pp. 112-120. doi: $10.3384 /$ ecp 18154112

[12] Modelica - A Unified Object-Oriented Language for Physical System Modeling: Language Specifications, Available at https://www.modelica.org/documents/ModelicaSpec32Revision2.pdf

[13] Siemens AG. (2019). PSS/E-high-performance transmission planning and analysis software. Retrieved from: https://goo.gl/xPFpjf

[14] F. J. Gómez, M. Aguilera Chaves, L. Vanfretti and S. H. Olsen, "Multi-Domain Semantic Information and Physical Behavior Modeling of Power Systems and Gas Turbines Expanding the Common Information Model," in IEEE Access, vol. 6, pp. 72663-72674, 2018. doi: 10.1109/ACCESS.2018.2882311 